\title{
APPLYING PDC FOR THE RECOGNITION OF FIREARM'S CALIBRE
}

\author{
Rafael Rodrigues Mendes Ribeiro*, Talysson Manoel de Oliveira Santos*, Tadeu Júnior \\ Gross*, Jordão Natal de Oliveira Júnior*, Victor Hugo Batista Tsukahara*, Carlos \\ DiAS MACIEL*
}

*Signal Processing Laboratory, Department of Electrical and Computing Engineering, São Carlos School of Engineering, University of São Paulo, São Carlos, São Paulo, Brazil

Emails: rafael.mendes.ribeiro@usp.br, talyssonsantos@usp.br, tadeu.gross@alumni.usp.br, jordao.oliveira@usp.br, vhbtsukahara@usp.br, carlos.maciel@usp.br

\begin{abstract}
Nowadays, there has been an enormous increase in audio recordings, some of these of the interest of audio forensics. Audio forensics has been used in many different ways for recognising firearm's calibres. In this work, we use Partial Directed Coherence (PDC) on recordings of capsules hitting the ground to recognise the firearm's calibre. Six different calibres were recorded in a controlled environment, and these audios were cut to have the same size. These audios were analysed using PDC. The results show that the audios of the calibres are orthogonal; thus, for this experiment being impossible to get a false positive while using the proposed methodology.
\end{abstract}

Keywords - Calibre, Recognition, PDC

\section{Introduction}

Nowadays, handheld devices capable of multimedia recording are everywhere, above all in the form of smartphones. That quasi ubiquity comes generating a significant amount of audio recordings, some of them inevitably of forensic interesting - for example, an unpretentious audio recording that timely registers a pistol shot followed by a tinkling in the floor of the ejected cartridge.

Audio forensics refers to the analysis, acquisition and evaluation of audio recordings admissible to a court of law as forensic evidence. It is typically used to verify the authenticity and integrity of audio evidence, enhancement of audio recording improving speech intelligibility and audibility of low-level sounds, and speaker identification (Khan et al., 2018; Zakariah et al., 2018).

Despite the fact that these are the primary uses of audio forensics, with the increase of use of firearms in crimes (Manley et al., 2019; Maher, 2007) (in Brazil the increase was of $27.4 \%$ from 2006 to 2016 (Ipea, 2018)) and in the military in the last few years combined with the increase of audio recordings with crimes (Larrain et al., 2015) the techniques of audio forensics adapted to new types of scenarios. Thus, recently, several acoustical detection, location and classification systems for gunshot sounds (Maher, 2007) have been developed. These systems usually use the muzzle blast, or the acoustical shock wave or both (Thumwarin et al., 2014; Mäkinen e Pertilä, 2010; Maher, 2006) as the basis of its analysis. One example of these systems is presented in the work of Thuwarin et al. (2014), where they use the gunshot blast to identify the calibre of the firearm by recognising the gum. A different example would be the work of Mäkinen and Pertilä (2010) in which they use the firing sound and the shock wave to estimate the shooter location and the bullet trajectory, calibre and speed.

Going through the road not taken, Larrain et al. (2015) created a system to identify the firearm calibre using the acoustical signature of the ejected cartridge case inspired by acoustic physics concepts, such as resonance of the capsule. They applied their system to audios of four different calibres in a controlled environment and 339 gunshots recordings extracted from YouTube and obtained a $75 \%$ rate of precision of identification. Following their lead, in this work, the Partial Directed Coherence (PDC) was used to recognise the calibre of the firearm by its capsule sound on hitting the ground.

This paper is organised as follows, in Section 2 the theory behind our method is presented, in Section 3 the procedure of the analysis and the signal acquirement is described in detail, in Section 4 the results are shown and discussed and, finally, in Section 5 the conclusions are presented.

\section{Theory}

In this section, the theory behind this work is approached on the following subsections.

\subsection{Partial Directed Coherence (PDC)}

Partial Directed Coherence is a frequency-domain approach to describe the direction of information flow between multivariate time signals using the decomposition of multivariate partial coherences computed from autoregressive models (Baccalá e Sameshima, 2001). This concept is a frequencydomain approach of Granger Causality. 
Given a set of $N$ signals $\mathbf{X}[n]=\left[\mathbf{x}_{1}[n], x_{2}[n]\right.$, $\left.\ldots, x_{N}[n]\right]$, the $\mathrm{AR}$ representation is

$$
\mathbf{X}[n]=\sum_{r=1}^{p} \mathbf{A}_{r} \mathbf{X}[n-r]+\mathbf{E}[n]
$$

being $p$ the order of autoregressive equation, $A_{r}$ representing the coefficients matrix and $E[n]$ the noise matrix. The $a_{i j}$ elements of $\mathbf{A}_{\mathbf{r}}$ represents the linear interaction effect of $x_{j}(n-r)$ towards $x_{i}(n)$.

The coefficients matrix $\mathbf{A}_{\mathbf{r}}$ is transformed into $\mathbf{A}(f)$ by

$$
\mathbf{A}(f)=\sum_{r=1}^{p} \mathbf{A}_{\mathbf{r}} e^{-i r 2 \pi f},
$$

and the joint spectral density is obtained by

$$
\overline{\mathbf{A}}^{-1}(f)=(\mathbf{I}-\mathbf{A}(f))^{-1}
$$

The PDC from $j$ to $i$ is given by

$$
\bar{\pi}_{i j}(f)=\frac{\bar{A}_{i j}(f)}{\sqrt{\overline{\mathbf{a}}_{j}^{H}(f) \overline{\mathbf{a}}_{j}(f)}},
$$

where $\bar{A}_{i j}(f)$ is the $i, j$ th element of $\overline{\mathbf{A}}(f)$. Equation 4 represents the relative interaction strengths between signals if adopting the following normalization properties hold:

$$
0 \leq\left|\bar{\pi}_{i j}(f)\right|^{2} \leq 1
$$

and

$$
\sum_{i=1}^{N}\left|\bar{\pi}_{i j}(f)\right|^{2}=1,
$$

for all $1 \leq j \leq N$.

\subsection{Hilbert Space}

A Hilbert Space is a complete pre-Hilbert space, where a pre-Hilbert space is a complex vector space $\mathscr{P}$ in which for each pair of vectors $x, y$ of $\mathscr{P}$ there is a scalar product $(x \mid y)$ of $x$ and $y$ that is a complex number and obeys these rules:

1. $(y \mid x)=(x \mid y)^{*}$

2. $(x+y \mid z)=(x \mid z)+(y \mid z)$

3. $(\lambda \mid y)=\lambda(x \mid y)$

4. $(x \mid x)>0$ when $x \neq \theta$

where $\theta$ is the zero vector and $x$ and $y$ can be functions having scalar values defined on a set $\mathscr{J}$ where $x=y$ when $x(t)=y(t), \forall t \in \mathscr{J}$, and the functions $\theta,-x, x+y$ and $\lambda x$ are defined by the formulas:

$$
\text { 1. } \theta(t)=0
$$

2. $(-x)(t)=-x(t)$

3. $(x+y)(t)=x(t)+y(t)$

4. $(\lambda x)(t)=\lambda x(t)$

thus being an vector space of scalar-valued functions on $\mathscr{J}$ (Berberian, 1961).

\section{Material and Methods}

This work can be divided into three parts: audio acquisition, pre-processing and analysis. Considering that, the following subsections describe in detail the evaluation of the proposed methodology and how each of these three parts was done.

\subsection{Resources}

The algorithms were implemented using a Jupyter Notebook interface for Python3. Scientific computation packages such as Numpy (Oliphant, 2006), Matplotlib (Hunter, 2007) and Connectivipy (Krzemiński e Kamiński, 2017) aid the algorithms.

The codes were run on a notebook with Intel (R) Core i3 M350 2.27GHz processor, 4Gb RAM and Linux Lite 4.0 operating system.

\subsection{Simulation}

To evaluate and exemplify, the proposed methodology was applied to analyse two signals $x_{1}(n)$ and $x_{2}(n)$ given by the equations 7 and 8 respectively

$$
\begin{aligned}
x_{1}(n)= & 0.9 x_{1}(n-1)-0.5 x_{1}(n-2)+e_{1}, \\
x_{2}(n)= & 0.8 x_{2}(n-1)-0.5 x_{2}(n-2)+ \\
& 0.16 x_{1}(n-1)-0.2 x_{1}(n-2)+e_{2},
\end{aligned}
$$

with $\operatorname{var}\left(e_{1}\right)=1, \operatorname{var}\left(e_{2}\right)=0.7$ and $\operatorname{cov}\left(e_{1}, e_{2}\right)=$ 0.4 .

\subsection{Acquisition}

The audio acquisition was made in a controlled indoor environment. The room was $3 \mathrm{~m} \times 3 \mathrm{~m}$. Each of the capsules was dropped from a $1 \mathrm{~m}$ height. Meanwhile, the handheld audio recorder was positioned at the height of $1.1 \mathrm{~m}$ from the ground. The used handheld recorder was the TASCAM DR-05X $\mathrm{X}^{1}$ with a sampling rate of 44100 samples/s. The capsules used where $.380,9 \mathrm{~mm}, .40, .45,5.56$ and 7.62 calibre. They were dropped in this order twice using the setup described.

\footnotetext{
${ }^{1}$ https://tascam.com/us/product/dr-05x/top
} 


\subsection{Pre-processing}

For the audio analysis, the audio had to be aligned to the start of the sound of the capsule. This way, the silence time before would not affect the analysis. The signals also needed to have the same length, to achieve that it a window size of 20.000 samples that was big enough to fit the first sound of the capsules was defined. This separation is shown in Figure 1.

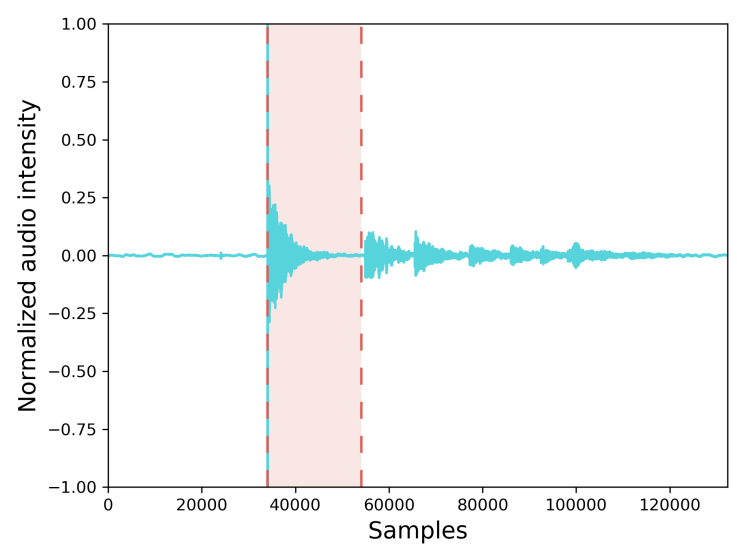

Figure 1: Plot of one of the capsules sound. The shaded region between the dashed lines is the region used for the analysis. This region was separated in the pre-processing.

\subsection{Analysis}

The analysis of the selected regions was done applying the PDC for the firsts audios of all the six capsules. After that, the PDC was applied for the second audio of the capsules comparing with the first audios.

\section{Results and Discussion}

The PDC of the theoretical signals used to evaluate the methodology is given in Figure 2.

Analysing the figure 2, it becomes clear that there is an interaction from $x_{1}$ to $x_{2}$, but there is not an interaction from $x_{2}$ to $x_{1}$. In addition to that, the results show strong interaction from $x_{1}$ to $x_{1}$ and from $x_{2}$ to $x_{2}$, which was expected.

For the PDC of all the firsts audios of all the six capsules, Figure 3 was obtained. From it, it is clear that no capsule calibre has an effect on another on both ways, thus showing us that if you consider the sounds of the capsules as vectors in a Hilbert Space these vectors are orthogonal, which means that it is impossible to have a false positive on the identification using PDC.

The results for the PDC of two of the second audios with one of the first can be seen in Figures 4 and 5. This result confirms what was noted on the previous result that using $\mathrm{PDC}$, it is impossible to

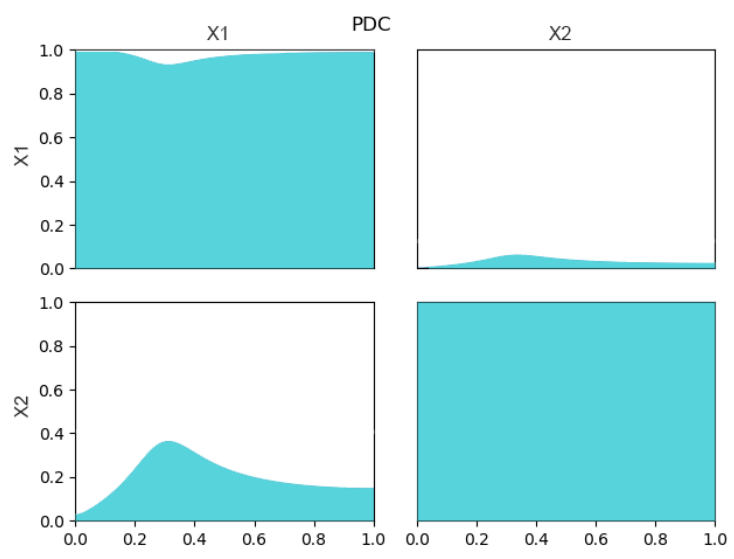

Figure 2: Example of PDC of the signals $x_{1}$ and $x_{2}$.

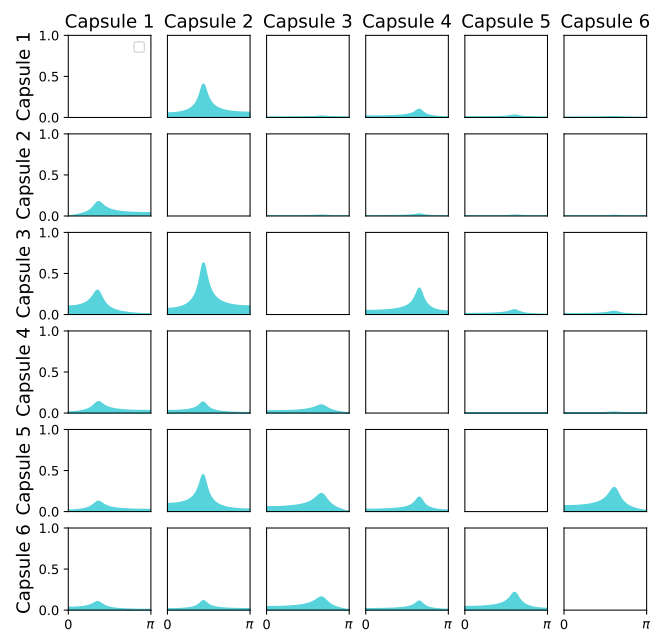

Figure 3: Result of the PDC applied to the firsts sounds of the capsules.

misjudge, which is the calibre of the capsule as the $\mathrm{PDC}$ is only maximum to the correct capsule.

\section{Conclusion}

From the results obtained, it is possible to conclude that the analysis using PDC is very useful for the identification of bullet calibre by the sound of the capsule hitting the ground.

\section{Acknowledgements}

The FAPESP (Fundação de Amparo à Pesquisa do Estado de São Paulo) for the financial support, process 2018/23139-8.

\section{References}

Baccalá, L. A. e Sameshima, K. (2001). Partial directed coherence: a new concept in neural 

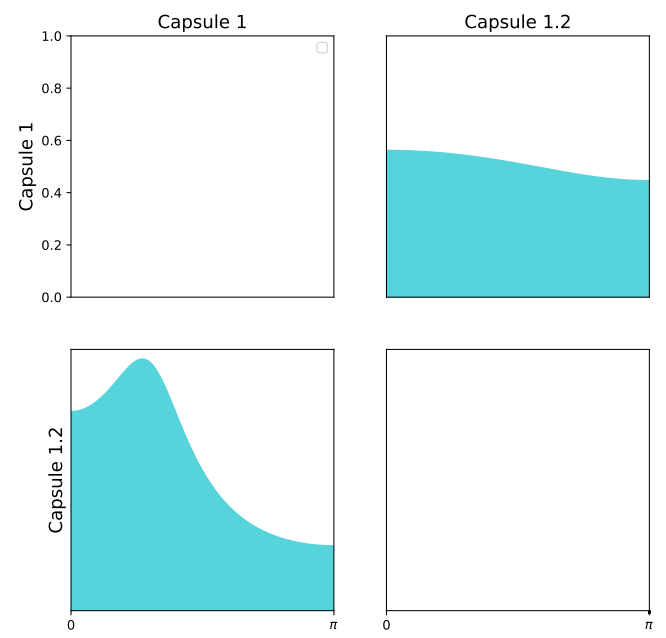

Figure 4: Result of the PDC applied to the first sound of capsule one and the second sound of capsule one.
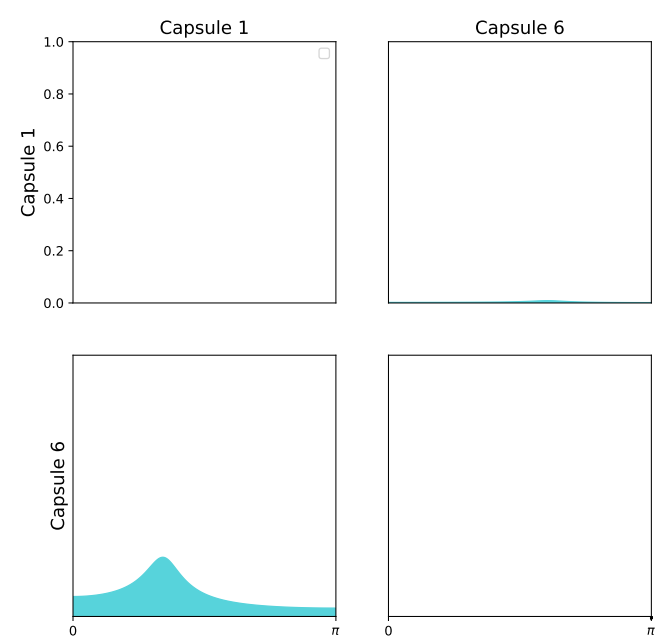

Figure 5: Result of the PDC applied to the first sound of capsule one and the second sound of capsule six.

structure determination, Biological Cybernetics 84(6): 463-474.

Berberian, S. K. (1961). Introduction to Hilbert space, Oxford University Press.

Hunter, J. D. (2007). Matplotlib: A 2d graphics environment, Computing in Science $\mathcal{E}$ Engineering 9(3): 90-95.

Ipea (2018). Atlas da Violência 2018.

Khan, M. K., Zakariah, M., Malik, H. e Choo, K.K. R. (2018). A novel audio forensic data-set for digital multimedia forensics, Australian Journal of Forensic Sciences 50(5): 525-542.
Krzemiński, D. e Kamiński, M. (2017). Connectivipy documentation. [Online; accessed: 2019-08-14].

URL: 1

Larrain, L. V. G. L., da Costa, J. P. C. L. e Gross, T. J. (2015). Identification of firearm calibers via acoustical signature of the ejected cartridge case, 2015 9th International Conference on Signal Processing and Communication Systems (ICSPCS), IEEE, pp. 1-6.

Maher, R. C. (2006). Modeling and signal processing of acoustic gunshot recordings, 2006 IEEE 12th Digital Signal Processing Workshop 8 4th IEEE Signal Processing Education Workshop, IEEE, pp. 257-261.

Maher, R. C. (2007). Acoustical characterization of gunshots, 2007 IEEE Workshop on Signal Processing Applications for Public Security and Forensics, IEEE, pp. 1-5.

Mäkinen, T. e Pertilä, P. (2010). Shooter localization and bullet trajectory, caliber, and speed estimation based on detected firing sounds, Applied acoustics 71(10): 902-913.

Manley, N. R., Croce, M. A., Fischer, P. E., Crowe, D. E., Goines, J. H., Sharpe, J. P., Fabian, T. C. e Magnotti, L. J. (2019). Evolution of firearm violence over 20 years: integrating law enforcement and clinical data, Journal of the American College of Surgeons

Oliphant, T. (2006). NumPy: A guide to NumPy, USA: Trelgol Publishing. [Online; accessed: 2019-05-20].

URL: 1

Thumwarin, P., Wakayaphattaramanus, N., Matsuura, T. e Yakoompai, K. (2014). Audio forensics from gunshot for firearm identification, The 4th Joint International Conference on Information and Communication Technology, Electronic and Electrical Engineering (JICTEE), IEEE, pp. 1-4.

Zakariah, M., Khan, M. K. e Malik, H. (2018). Digital multimedia audio forensics: past, present and future, Multimedia tools and applications 77(1): 1009-1040. 